\title{
Les Peines et Leurs Attenuations en Droit Penal Congolais
}

\author{
Kaciunga Mbenga Joseph ${ }^{1}$
}

\section{Résumé}

La justice pénale congolaise soumet à une rude épreuve, l'effectivité de l'Etat de droit. Il se dégage une quasi-totale inadéquation entre le service de maintien de l'ordre qu'il doit offrir à la société et la rééducation des criminels qui sont membres de la société dont-il doit protéger. Pour maintenir l'ordre social, l'Etat recourt à l'application des peines qui sont régit par le principe de la légalité des peines. Sans doute, le droit pénal est de stricte interprétation et application. Cependant, l'Etat, reconnaissant que le droit pénal doit être humanisé, a prévue les circonstances atténuantes. En droit positif congolais les circonstances atténuantes constituent l'assouplissement du principe de la légalité et non sa violation. En affirmant la liberté du juge dans l'appréciation des éléments constitutifs fondant la rétention des excuses atténuantes, le législateur devrait limiter son pouvoir dans la fixation du taux de la peine, en indiquant le minimum en deçà du quel il ne peut descendre.

Mots- clés : peines -circonstances- atténuantes- droit congolais.

\begin{abstract}
The Congolese criminal justice system is putting the effectiveness of the rule of law to a severe test. There is an almost total inadequacy between the policing service it must provide to society and the rehabilitation of criminals who are members of the society it must protect. To maintain social order, the State uses the enforcement of sentences, which are governed by the principle of the legality of sentences. Undoubtedly, criminal law is strictly interpreted and applied. However, the State, recognizing that criminal law must be humanized, has provided for mitigating circumstances. In Congolese positive law, mitigating circumstances constitute the relaxation of the principle of legality and not its violation. By affirming the judge's freedom to assess the constituent elements on which to base the retention of mitigating excuses, the legislator should limit his or her power to set the rate of the sentence, indicating the minimum below which he or she may not go under it.
\end{abstract}

\section{INTRODUCTION}

La peine est définie comme un mal infligé à titre de punition par le juge à celui qui est reconnu coupable d'une infraction ${ }^{2}$. En vue de maintenir l'ordre public, la puissance publique

1 Avocat au Barreau de Kinshasa Gombe. kaciungajoseph@gmail.com.

2 NYABIRUNNGU mwene SONGA, Traité de droit Pénal Général Congolais $2_{2} 2^{2 \mathrm{me} e}$ éd. Kinshasa, 2007, P. 31. 
y recourt souvent. Dans l'application de la peine, l'individu risque ce qu'il a de plus cher, entre autres : la vie, la liberté et même les biens, car étant considéré comme dangereux pour la société. Aucune société n'est parfaite, exempte des délinquants, l'Etat doit par l'application de la peine prendre en compte le caractère humain de ses sujets en appliquant les circonstances atténuantes dans la condamnation du délinquant. Nous ne saurons accorder du crédit à l'application rigoureuse de la peine qui va jusqu'à supprimer l'infracteur qui du reste, demeure un élément de l'existence de la société dont l'Etat protège. Sachant que la peine joue tant des fonctions dans le maintient de l'ordre public, notre question de départ s'énonce comme suit : l'application des circonstances atténuantes, permet-elle à la peine de remplir ses fonctions? Notre propos s'articulera ainsi autour de trois points : le premier portera sur les peines en droit positif congolais; le second examinera les circonstances atténuantes en droit pénal congolais et le troisième traitera la problématique de l'incidence des circonstances atténuantes sur les fonctions de la peine. Je viens ainsi de tracer le plan de cet article qui comportera trois grands points.

\section{A. LES PEINES EN DROIT POSITIF CONGOLAIS}

La commission d'une infraction brise le pacte social conclu implicitement entre les citoyens et l'Etat ${ }^{3}$. Comme l'époque de la justice privée et celle de la vengeance privée sont révolues; désormais, c'est à l'Etat qu'appartient le monopole de la répression. Il s'y apprend par l'action publique définie comme celle qui est portée devant une juridiction répressive en vue de l'application des peines à l'auteur d'une infraction ${ }^{4}$. La peine en droit positif congolais obéit à plusieurs principes ${ }^{5}$ mais surtout celui de la légalité ${ }^{6}$ énoncé par le dicton « NULLA POENA, SINE LEGE » c'est à dire pas des peines sans textes de loi. Ce principe nous rend curieux de découvrir le cadre juridique des peines en droit congolais.

\section{CADRE JURIDIQUE}

L'actuelle loi fondamentale de la République Démocratique du Congo ${ }^{7}$ consacre le principe de la légalité des peines en ces termes : « il ne peut être infligé de peine plus forte que celle applicable au moment où l'infraction est commise » par ce principe, l'Etat Congolais ne consacre pas seulement la non rétroactivité des peines, mais aussi une garantie contre l'ar-

3 GERARD LOPEZ et STAMATOS TZITZIS, Dictionnaire des sciences Criminelles, Paris, 2004 P.542

4 SERGE GUINCHARD et THIERY DEBARD (Dir), lexique des termes juridiques, Paris, 2012, P 31.

5 La peine obéit aux principes d'égalité, de dignité, de la légalité etc.

6 Article $1^{\mathrm{er}}$,du Décret du 30 Janvier 1940 portant code pénal congolais tel que modifié à ce jour, in J.O, $45^{\text {ème }}$, Numero Spécial 30 Novembre 2004.

7 L'article 17 al.5 la constitution du 18 Février 2006 telle que révisée par la loi № 11/002 du 20 Janvier 2011 portant révision de certains articles de la constitution de la RDC, J.O. RDC, $52^{\text {eme }}$ Année, numéro spécial, 5 février 2005. 
bitraire du juge d'une part, et d'autre part, émet une protection contre la violation de l'article 23 du statuts de la cour pénale internationale.

En droit pénal ordinaire les peines sont portées par les dispositions de l'article $5 \mathrm{du}$ code pénal congolais qui dispose : « les peines applicables aux infractions sont:

1. La mort;

2. Les travaux forcés;

3. La servitude pénale;

4. L'amende;

5. La confiscation spéciale;

6. L'obligation de s'éloigner de certains lieux ou d'une certaines région;

7. La résidence imposée dans un lieu déterminée;

8. La mise à la disposition de la surveillance du gouvernement $»^{8}$.

Cette énumération n'est plus faite pour le formalisme juridique, mais dans l'ensemble, les peines jouent tant des fonctions dont nous sommes censés découvrir.

\section{LES FONCTIONS DE LA PEINE}

Bien que la peine soit inséparable de l'idée de souffrance ${ }^{9}$ la peine n'inspire pas seulement la peur d'une contrainte physique mais bien plus, la sanction pénale est censée remplir plusieurs fonctions ${ }^{10}$ entre autres la fonction rétributive, préventive ainsi que celle éliminatrice. Il sied d'analyser chacune des fonctions pour en comprendre le sens.

\section{LA FONCTION RETRIBUTIVE}

Chaque société a ses valeurs qu'aucun individu ne peut violées et resté impuni. Un proverbe Grec ne dit-il pas « qu'il convient de maîtriser la barbarie de l'être humain pour rendre la vie douce?» ${ }^{11}$ Cette fonction vise à faire payer le délinquant pour le mal infligé à la société par son acte criminel. Lorsqu'un délinquant commet une infraction, il contracte une dette envers la société et il doit la payer. C'est en cela qu'une peine demeure une rétribution car le sentiment comme l'expression populaire sont que "justice est faite" lorsque l'auteur du crime crapuleux monte à l'échaude. ${ }^{12}$ Cette fonction constitue un soulagement pour la société.

8 Article 5 du décret du 30 Janvier 1940 portant code Pénal Congolais tel que modifié à ce jour, in J.O $45^{\text {ème }}$ numéro Spécial 30 Novembre 2004.

9 NYABIRUNGU MWENE NSONGA, Droit pénal Général Zaïrois, Kinshasa, 1989, P. 294.

10 KIFWABALA TEKILAZAYA, DEF FATAKI, MARCEL WETSH-OKO (Dir), la République Démocratique du Congo le secteur de la justice et l'Etat de droit in open society foundations, juillet 2013.

11 KATAMBWE MALIPO, la peine de mort en droit Criminel Congolais : Nécessité ou nachronisme au regard du Statut de Rome, in les analyses juridiques $N^{\circ}$ 1/2004, Lubumbashi, P. 21.

12 BOUZA ET PINATEL, cité par NYABIRUNGU MWENE SONGA, note 8, P. 295. 


\section{LA FONCTION PREVENTIVE}

Cette fonction atteint les deux pôles, d'une part, elle concerne le délinquant c'est-à-dire la prévention individuelle (a) et d'autre part, elle prévient la société, donc la prévention générale (b)

a) la fonction de prévention individuelle

Avec cette prévention spéciale, on considère que l'infracteur qui a purgé sa peine connait sans faille les désagréments qu'elle comporte. Le délinquant aura peur de récidiver, c'est la fonction utilitaire au sens BENTHAMIEN : l'agent doit avoir plus d'intérêt à respecter la loi qu'à la violer ${ }^{13}$, et donc, la peine joue le rôle d'intimidation spéciale.

\section{b) La fonction de prévention générale}

La peine joue un rôle d'avertissement en l'endroit de la société. Une fois appliquée au délinquant, elle constitue une mise en garde adressée à la société. C'est ainsi lorsque les crimes spectaculaires sont commis, l'Etat par le principe de litinérance, tient des audiences foraines en vue de dissuader le reste de la population à commettre les mêmes actes. Dans ce même but, les audiences sont souvent publiques.

\section{c) La fonction éliminatrice}

La fonction d'élimination met le délinquant hors d'état de nuire, toutes les peines jouent ce rôle mais, celle de mort remplit cette fonction d'une manière excellente d'autant plus que la légalité de la peine de mort n'est pas aujourd'hui contestée dans notre droit quels que soient les arguments utilisés pour l'abolition de cette peine par certains penseurs ${ }^{14}$.

D'une manière générale l'application de la peine doit tenir compte non seulement de la vie de la société, mais aussi de celle du délinquant. Le droit pénal ne doit pas être considéré comme un droit robotique mais, il serait judicieux de l'humaniser en prenant en compte plusieurs facteurs qui ont concouru à la commission d'infraction.

\section{B. LES CIRCONSTANCES ATTENUANTES EN DROIT PENAL CONGOLAIS}

A l'instar des autres législateurs du monde, le législateur congolais n'a pas défini ce qu'il faut entendre par les circonstances atténuantes, il se limite à édicté la modalité d'application. C'est à la disposition de l'article 18 alinéa 1 et 2 du code pénal congolais que le législateur prévoit la modalité d'application en ces termes «s'il existe des circonstances atténuantes, la peine de mort pourra être remplacée par la servitude pénale à perpétuité ou par 
une servitude pénale dont le juge déterminera la durée. Les peine de servitude pénale et d'amende pourront être réduite dans la mesure déterminée par le juge » mais c'est la doctrine qui s'accorde pour dire que les circonstances atténuantes sont des particularités, non déterminées par loi, qui accompagnent une infraction et dont l'effet, lorsque leur existence est reconnue, est de permettre au juge d'atténuer la peine. ${ }^{15}$ De l'analyse minutieuse de cette définition, il se dégage quelques assertions dont nous devons comprendre à savoir : l'autorité d'appréciation (I) des éléments constructifs des circonstances atténuantes (II) ainsi que le but des excuses atténuantes (III).

\section{L'AUTORITE D'APPRECIATION}

Les peines prévues par la loi sont parfois fixe mais le plus souvent variables entre un minimum et un maximum ${ }^{16}$ c'est à la juridiction de déterminer le taux de la peine, voilà pourquoi le professeur NYABIRUNGU mwene SONGA martèle que dès qu'il a constaté l'existence de l'infraction dans tous ses éléments objectifs, qu'il est convaincu de sa culpabilité, le juge n'est pas encore au bout de ses peines. Encore doit-il déterminer la sanction la plus appropriée, compte tenu de l'acte et du délinquant ${ }^{17}$. Sur pieds des articles 18 et 19 du code pénal congolais, les cours et Tribunaux sont investis du pouvoir d'apprécier des particularités qui fondent des situations atténuantes et donc, c'est le juge qui a le pouvoir. Bien que le juge en apprécie souverainement; il y a des limites qui lui sont imposées dans la modalité d'application, en ce qu'il ne sera pas prononcé, toutefois des peines de servitude pénale de moins d'un jour, ni de peine d'amende de moins d'un Zaïre d'une part et d'autre, tout jugement admettant des circonstances atténuantes les indiquera et les énuméra ${ }^{18}$. Autrement dit, le juge ne peut accorder des excuses atténuantes sans les motiver. Cependant, malgré ces limitations quant à l'application, le juge apprécie les éléments qui concourent à la rétention des circonstances atténuantes souverainement.

\section{LES ELEMENTS CONSTRUCTIFS DES CIRCONSTANCES ATTENUANTES}

Nous sommes d'avis que la première conséquence du principe de la légalité des incriminations et des peines, c'est l'interdiction faite au juge d'imaginer et de suppléer aux textes répressifs ${ }^{19}$. Cependant, nous devons comprendre que le droit pénal ne doit pas être robotique mais plutôt humanisé. Sachant que les excuses atténuantes sont facultative et personnelle et que le juge peut donc les trouver dans n'importe quel élément même postérieur à l'infrac-

15 EMILE BRUNET, JEAN SERVAIS, CHARLES RESTEAU (Dir), Répertoire pratique du droit Belge, T II Bruxelles P. 48.

16 GEORGES LEVASSEUR et JEAN PIERRE DOUCET,_le Droit Pénal Appliqué,_Paris, P. 294.

17 NYABIRUNGU MWENE SONGA, note 8, P. 318.

18 Articles 17 et 18 du code pénal congolais.

19 ESIKA MAKAMBO ESO BINA, Le code Pénal Zaïrois annoté, Lubumbashi, 1977, P 18. 
tion qu'il en affirme l'existence de façon discrétionnaire ${ }^{20}$.Nous avons eu à approcher quelques juges dont nous avons garantie l'anonymat dans la présentation de leur propos tout en leur attribuant les surnoms en usant l'entretient semi directif ${ }^{21}$.

C'est ainsi, de notre entretien avec le juge CLERON, il nous dévoile que : « les circonstances atténuantes sont indéfinies, nous pouvons les trouver dans la personnalité du délinquant. Si nous constatons qu'il n'a jamais eu des antécédents judiciaires c'est à nous d'apprécier $»^{22}$ au juge Mado de renchérir que : « nous sommes les humains, nous pouvons trouver des éléments pouvant fondés notre conviction à retenir les circonstances atténuantes dans l'histoire liée au comportement de l'infracteur, dans le fait même ou encore dans l'environnement qui a occasionné la commission dudit fait, nous jouons sur base des éléments en présence $»^{23}$. De ces deux entretiens, nous remarquons que le juge, bien qu'étant souverain dans l'appréciation de fait, il part des éléments probant pour aboutir à la rétention des excuses atténuantes. Il se fonde soit sur la personnalité du délinquant, soit sur les faits même. C'est ainsi qu'on admet comme circonstances atténuantes : le peu de gravité de l'infraction, l'absence ou la ténuité du préjudice, la réparation du dommage causé, l'entrainement de la pression de la misère, les bons antécédents, les aveux, le repentir, etc... ${ }^{24}$,voila pourquoi nous soutenons que la notion... Des circonstances exceptionnelles qui peuvent faire réduire la peine en dessous du taux habituel correspond à une réalité ${ }^{25}$. on peut lire dans l'œuvre du juge Pénal que dans l'application de la peine à infliger à la citée, le tribunal aura égard au fait qu'elle est délinquant primaire, qu'elle est mère et grand-mère de famille mais aussi son état de santé et son âge avancé ne permettront pas qu'elle supporte les effets d'un emprisonnement, sur ce, le Tribunal la condamnera avec admission des larges circonstances atténuantes à un mois de servitude pénale avec sursis et ce, conformément à l'article $42 \mathrm{du}$ code pénal livre premier. ${ }^{26}$ Cette décision influence positivement l'application de la peine et donc le but est atteint savoir la réduction de la peine et surtout son individualisation. Même si une infraction est commise en corréité, le juge ne peut accorder les circonstances sans tenir compte des particularités de chacun des infracteurs. Un jugement ou arrêt peut contenir les circonstances atténuantes pour les uns et non pour les autres, voir même contenir les excuses exceptionnelles ainsi que aggravantes. Ces quelques éléments ne fixent

20 Crim 21 Novembre 1962 Bull N³34 par LEVASSEUR et DOUCET, note 15, P. 297.

21 C'est un entretien qui n'est ni entièrement ouvert, ni canalisé vers un grand nombre de question, mais qui laisse la liberté de la parole à l'interviewé lire à ce sujet R. KIENGE KIENGE INTUDI, initiation à la_Recherche Scientifique, Syllabus, Faculté de Droit, UNIKIN 2009-2010, P. 61.

22 Entretient du 18 et 19 du 4 juin 2018 avec un juge du Tribunal de Grande Instance de Kinshasa/ KALAMU.

23 Entretien réaliser le 06/2018 avec la Dame juge du Tribunal de paix de Kinshasa/Lemba.

24 EMILE BRUNET JEAN SERVAIS, CHARLES RESTEAU (Dir) note 14. P 48.

25 PIRON PIERRE, Codes et Lois du Congo Belge, $7^{\mathrm{èm}}$ éd., Bruxelles 1954, P.278.

26 Tribunal de paix de Kinshasa/Matete 21 Novembre 2017, sous RP 30 595/IV (Affaire NYANGA MBOMBO LAETITIA et Consort C/ MUJINGA KATOMBO HORTENCE) inédite .14 ${ }^{\mathrm{ème}}$ Feuillet. 
pas la peine mais, entrent en ligne de compte, influençant ainsi la conviction du juge pour retenir les circonstances atténuantes.

\section{BUT DES CIRCONSTANCES ATTENUANTES}

Les circonstances atténuantes ne visent pas seulement d'atténuer la peine mais aussi l'individualisation de la peine. Le châtiment doit correspondre au comportement du sujet. confirmer que les excuses atténuantes n'ont pour but que la réduction de la peine, rend formalisme l'application de la peine même. Cette question mérite un égard approfondit.

\section{L'INCIDENCE DES EXCUSES ATTENUANTES SUR LES PEINES}

La peine constitue depuis la plus haute antiquité la forme primitive de la réaction de l'autorité sociale aux agissements qui troublent l'ordre établi ${ }^{27}$. La peine à des caractères fondamentaux qui corroborent avec les buts qu'elle poursuit. La question épineuse est celle de savoir si les objectifs des peines seront atteint en cas d'application des circonstances atténuantes? les éléments de réponse s'articuleront autour de l'influence que peut avoir la rétention des circonstances atténuantes sur le principe de la légalité des peines (I) ainsi que sur l'application proprement dite des peines (II) avant que nous abordions les perspectives d'avenir (III)

\section{LA LEGALITE DES PEINES A L'EPREUVE DES CIRCONSTANCES ATTENUANTES}

Le principe de la légalité des délits et des peines comporte tant des conséquences, outres la non rétroactivité des peines; il consacre l'interdiction de l'analogie dans l'application des lois pénales. A cause de cette dernière conséquence, certains ont trouvé le principe de la légalité des incriminations et des peines insuffisant, voir dangereux pour la défense sociale $^{28}$. Ils ont fait observer que ce principe présente l'inconvénient de ne pas permettre l'individualisation de la peine et surtout d'assurer l'impunité aux malfaiteurs habiles qui savent profiter des lacunes de la loi ${ }^{29}$.

Nous constatons qu'en droit pénal congolais contrairement au droit Français et Belge, la rétention des circonstances atténuantes ne dénature pas l'infraction ${ }^{30}$ mais plutôt la peine. Le juge qualifiera sans faille le fait infractionnel, mais c'est le taux de la peine qui sera revu. Cela ressort de l'analyse du jugement rendu sous RMP 16423 par le tribunal de grande Instance de Kinshasa/Kalamu dans lequel, le prévenu fut initialement poursuivit pour l'extorsion mais, le juge requalifie le fait et dit établie en faits comme en droit l'infraction de vol à l'aide de violence prévue et punie à l'article 82 du code pénal congolais. Quant à la

27 GEORGES LEVASSEUR et JEAN PIERRE DOUCET, note 15, P.262.

28 ESIKA MAKAMBO ESO BINA, note 18, P. 19.

29 Idem.

30 EMILE BRUNET, JEAN SERVAIS, CHARLES RESTEAU, note 14, PP. 48-53. 
qualification, ce jugement respect le principe de la légalité mais, au lieu d'appliquer la peine dans le minimum et maximum prévu de cinq à vingt ans, le juge pénal condamne le prévenu à 10 mois de servitude pénale, à cause de circonstance atténuants retenues à son égard ${ }^{31}$ et donc, c'est la peine qui est flexible aux circonstances atténuantes.

La vraie incidence des excuses atténuantes, c'est l'assouplissement positif de la loi pénale. Nous concluons ce point tout en étant d'avis avec le professeur NYABIRUNGU Mwene Songa que «...l'admission des circonstances atténuantes du moment qu'elles sont rationnelles et motivées, constitue un assouplissement heureux du principe de la légalité $»^{32}$

\section{L'INFLUENCE DES CIRCONSTANCES ATTENUANTES DANS L'APPLICATION DES PEINES}

Nous sommes à moitié d'accord avec le professeur BAYONGA Ba MEYA MUNA KIMVIMBA lorsqu'il martèle que lors de la prise de ces décisions les magistrats doivent maitriser leurs passions pour n'écouter que la voix de leurs consciences ${ }^{33}$. Pour être complet, ils doivent avoir à même temps la loi en esprit et devant eux pour n'est plus rendre des décisions non conforme à celle-ci. L'effet des circonstances atténuantes n'est pas le même selon l'infraction dont la personne poursuivie a été reconnue coupable. ${ }^{34}$ cette position est confirmée par la teneur des jugements pénaux rendus par le Tribunal de Grande Instance de Kinshasa/KALAMU sous RMP 14877 et RMP 17975. Dans ce deux ouvres, les juges disent établie en faits comme en droit la prévention de vol qualifié en retenant des circonstances atténuantes pour tous les deux mais, condamnent KABAMBI MUTOMBO RODRICK à 3 ans de servitude pénale et sous RMP 17975, ils condamnent également BASWA PALE JOSEPH à 10 mois de servitude pénale. Malgré la rétention des circonstances; la diminution de la peine se différent compte tenu des éléments appréciable. ${ }^{35}$

Relevons que la principale influence des excuses atténuantes c'est la diminution de la peine. En matière criminelle, la déclaration de circonstances atténuantes entraine obligatoirement une modification de la peine corporelle ou privative de la liberté, ... En cas de circonstances atténuantes, la peine normale du crime doit être réduite d'un degré au moins, les autres degrés d'atténuation sont facultatifs. ${ }^{36}$ En République Démocratique du Congo, c'est l'article 18 du code pénal Congolais qui porte cette modalité d'application des circons-

31 Tribunal de Grande Instance de Kinshasa/KALAMU, 14 septembre 2018 Registre des prononcés Consulté le 1 Avril 2019.

32 NYABIRUNGU MWENE SONGA, note 8 P. 44.

33 BAYONGA BA MEYA MUNA KIMVIMBA, l'indépendance de la magistrature en République du Zaïre, in annales de la facultés de de Droit, Vol 1, Kinshasa, 1972 P. 19.

34 GEORGES LEVASSEUR et JEAN PIERRE DOUCET, le note 15, P. 297.

35 Tribunal de grande Instance de Kinshasa Kalamu, jugements rendus le 28 janvier - 04 Mars 2019 respectivement sous RMP 17 975/I et RMP 14877/II (Affaires Ministère Public et MOTO MOSELE C/BASWA MUTOMBO RODRICK inédit.

36 EMILE BRUNET JEAN SERVAIS, CHARLES RESTEAU (dir) note 14. P. 54. 
tances atténuantes à des peines et donc, le juge doit descendre graduellement d'un degré dans l'échelle des peines. Cette diminution de la peine vise, elle aussi un objectif heureux qui rend l'application de la peine très efficace, savoir l'individualisation de la peine. Parmi les infracteurs, il y a toujours des différences tenant notamment à leurs caractères et si plusieurs personnes sont condamnées dans une même affaire, l'octroi des circonstances atténuantes doit être examiné séparément pour chacune d'elles ${ }^{37}$. En somme, les circonstances atténuantes n'empêchent pas les peines de remplir leurs fonctions, mais elles concourent à l'efficacité même des objectifs d'une peine. Le juge choisi avec l'application des excuses atténuantes, les peines en fonctions du trouble social causé par le délinquant, de sa personnalité et surtout du rendement qu'il attend de la dite sanction sans quitter l'échelle des peines prévues par la loi. Enfin nous devons préciser que les excuses atténuantes ne s'appliquent pas aux peines complémentaires en droit congolais ${ }^{38}$.

\section{LES PERSPECTIVES D'AVENIR.}

Notre conscience scientifique est éveillée par la définition des circonstances atténuantes donnée par le professeur NYABIRUNGU Mwene Songa dans sa partie terminale qui dit que : «...le juge a la faculté de tenir compte pour atténuer la peine au point de descendre en dessous du minimum légal jusqu'à un jour de servitude pénale ou 1 Zaïre d'amende $»^{39}$

Cela dévoile la liberté absolue qu'a le juge dans la fixation des peines en cas de la rétention des circonstances atténuantes. Notre propos va dans le sens de réviser le décret du 30 Janvier 1940 portant code pénal modifié et complété à ce jour. Puisqu'il est reconnu dans la séparation des pouvoirs, au législateur seul de pouvoir limiter la liberté des citoyens par des normes pénales et que la loi pénale doit être de stricte application; le juge, lui chargé d'appliquer, ne doit pas jouir d'une liberté exorbitante dans la fixation de la peine. Il faut mettre des bornes à cette indulgence; laisser les juridictions descendre aussi bas qu'elles veulent c'est risquer un nivellement qui n'est plus inspiré par l'intérêt public au mépris des valeurs permanentes sur lesquelles se trouve basée notre civilisation ${ }^{40}$. Position renforcé par l'analyse de la décision juridictionnelle rendue le 08 Mars 2019 par le Tribunal de Grande Instance de Kinshasa/KALAMU sous RMP 18 145/V. ${ }^{41}$

$38 A u$ regard du dispositif du jugement rendu en date du 12 mars 1994 sous RP 12 567/VIII par le Tribunal de paix de Kinshasa/Kasa Vubu (affaire Ministère public et Madame MALO MALO BOLONDO C/WWINA BOLONDO et la contrainte par corps de 14 jours prévue à l'endroit de WAWINA et NGOTO en cas de non-paiement des frais de justice; ne pourra subir la diminution pour le fait des circonstance atténuantes car, elle est une peine subsidiaire ou complémentaire.

39 NYABIRUNGU MWENE SONGA, note 8, P. 319.

40 ANTOINNE SOHIER cité par PIRON, note 24, P. 278.

41 Tribunal de Grande Instance de Kinshaa/KALAMU 08 Mars 2019 sous RMP 18 145/V (Affaire Ministère public et MUNZEMBA C/KAYABA MASUKA GLOIRE Inédit. 
En fait, Sieur KAYABA MASUKA Gloire est poursuivi et condamné pour l'infraction de viol d'enfant mineure, nous sommes sans ignorer que le viol sur mineur mérite une répression exemplaire en vue de pouvoir décourager les éventuels criminel mais, le juge constate que le prévenu est un délinquant primaire, parvient à le condamné à 4 ans de servitude pénale à la place de rester même dans la fourchette légale de peine prévue à l'article 170 du code pénal congolais et donc, cette décision ne contribue pas à la fonction préventive de la peine.

Le législateur congolais s'est limité seulement à la limitation du minimum et du maximum des peines. Cela ne suffit pas, le législateur doit pouvoir encadré la liberté de juge pas dans la rétention des éléments fondant les circonstances atténuantes, mais plutôt en indiquant clairement en deçà duquel taux le juge ne peut aller en cas de la rétention des circonstances atténuantes. Pour éviter ces genres des décisions comme celle rendue sous RMP 18145 par les juges du Tribunal de Grande Instance de Kinshasa/KALAMU

Outre l'encadrement de la liberté du juge, le législateur doit pouvoir amener la lumière quant à l'application de la peine des travaux forcés. En cas des circonstances atténuantes de la peine capitale, ce sont les travaux forcés qui sont retenus.

En cas des circonstances atténuante dans l'application de la peine des travaux forcés, jusqu'à qu'elle limite peuvent-ils être réduit? Peuvent-ils être remplacés par la servitude pénale de combien des jours? et comment doit-on en exécuter en cas des circonstances atténuantes? Toutes ces questions nous mettent en droit de proposer au législateur de pouvoir revoir même la nomenclature de cette peine.

\section{CONCLUSION}

Cet article ainsi fini a eu pour objet de rechercher l'incidence des circonstances atténuantes dans l'application des peines. Le fil conducteur était la question de savoir si les circonstances atténuantes une fois retenues, permettraient aux peines d'atteindre leurs fonctions? les éléments de la réponse ont démontré que l'application des circonstances atténuantes renforce même l'effectivité de la peine car, elles permettent au juge d'individualiser la peine c'est qui est un atout pour humaniser le droit pénal. Relevons tout de même que les excuses atténuantes constituent l'assouplissement du principe de la légalité des peines en laissant la marge au juge de pouvoir choisir, malgré le fait repréhensible, la peine qui s'adapte à la personnalité du délinquant. Ce faisant, le droit pénal devra resté un droit non robotique mais, un droit humanisé.

\section{BIBLIOGRAPHIE}

\section{A. TEXTES JURIDIQUES}

1. La constitution du 18 Février 2006 telle que modifiée et complétée par la loi № $11 / 002$ du 20 janvier 2011 portant révision de certains articles de la constitution de la RDC in J.O. RDC $52^{\text {ème }}$ numéro spécial Kinshasa 5 Février 2011. 
2. Traité de Rome du 17 juillet 1998 portant statut de la Cour Pénale Internationale.

3. Décret du 30 janvier 1940 portant Code Pénal Congolais tel que modifié à ce jour in J.O. RDC $45^{\text {ème }}$ numéro Spécial, 30 Novembre 2004.

\section{B. DOCTRINE}

1. NYABIRUNGU MWENE SONGA, traité de droit pénal général, 1 ère éd. Kinshasa, DES 1989.

2. LEVASSEUR G. et DOUCET J.P., le Droit Pénal Appliquée Paris, CUJA, S.D.

3. NYABIRUNGU MWENE SONGA, traité de Droit Pénal Général, $2^{\text {ème }}$ éd. Kinshasa, EUA, 2007.

4. BRUNET E, SERVAIS, J RESTAUX CH., Répertoire Pratique du Droit Belge T.II., Bruxelles, EEB, 1949.

5. ESIKA MAKAMBO ESOBINA, le Code Pénal Zaïrois Annoté, Lubumbashi,1977.

6. PIRON P. Codes et Lois du Congo Belge, $7^{\text {ème }}$ éd. Bruxelles, Larcier 1954.

1. GUINCHARD S. et DEBAR Th., Lexique des termes Juridiques, Paris, 2012.

2. LOPEZ G., et STAMATOS TZITZIS, Dictionnaire des sciences criminelles, Paris 2004.

3. KIFWABALA TEKILA ZAYA, DEF FATAKI, MARCEL WETSH-OKO La République Démocratique du Congo : Secteur de la Justice et l'Etat de Droit in Open Society Foundations, 2013.

4. KATAMBWE MALIPO, la peine de mort en droit Criminel Congolais : Nécessité ou nachronisme au regard du statut de Rome, in les analyse juridiques $\mathrm{N}^{\circ} 1$ Lubumbashi 2004.

5. BAYONGA BA MEYA MUNA KIMVIMBA, l'indépendance de la magistrature en République du Zaïre, in annales de la facultés de de Droit, Vol 1, Kinshasa, 1972.

\section{DECISIONS JUDICIAIRES}

1. R.M.P. 30595/IV/Tribunal de paix de Kinshasa/MATETE.

2. R.M.P. 16423/Tribunal de Grande Instance de Kinshasa/Kalamu

3. R.P. 12 567/VIII/Tribunal de Paix de Kinshasa/Kasa-Vubu

4. R.M.P. 17975/I/Tribunal de Grande Instance de Kinshasa/Kalamu

5. R.M.P. 148 77/II Tribunal de Grande Instance de Kinshasa/Gombe

6.nR.M.P. 18 145/V Tribunal de Grande Instance de Kinshasa/Kalaamu 